\title{
DECISION TREE CLASSIFICATION MODEL FOR DETECTING AND TRACKING PRECIPITATING OBJECTS FROM SERIES OF METEOROLOGICAL IMAGES
}

\author{
S. Ramirez ${ }^{\mathrm{a} *}$ I. Lizarazo $^{\mathrm{b}}$ \\ ${ }^{a}$ Facultad de ingenería, Universidad Distrital Francisco Jose de Caldas, Bogota DC., Colombia - seramirezf@correo.udistrital.edu.co \\ ${ }^{\mathrm{b}}$ Facultad de Ciencias Agrarias, Universidad Nacional, Bogota, DC., Colombia - ializarazos@ unal.edu.co
}

KEY WORDS: Mesoscale Convective Systems, Precipitating Objects, Classification Model, Decision Trees, Meteorological Images

\begin{abstract}
:
Accurate detection and identification of convective (cumulonimbus) clouds, which are potentially precipitating objects, as well as tracking cloud movement, are important tasks to locate and predict precipitation. In the present work, a Decision Tree classification model was used to locate and track precipitating objects from series of GOES-13 meteorological image sub-scenes covering the territory of Colombia, located to the northwest corner of South America. Results show that it is possible to infer a classification model that can be used repeatedly for accurately locating and tracking precipitating objects from multispectral meteorological images.
\end{abstract}

\section{INTRODUCTION}

Knowledge of convective systems' cloud processes and their evolution is important for understanding weather and climate, particularly over the tropics (Vila et al., 2008). In both equatorial and mid tropical latitudes, cumulonimbus $(\mathrm{Cb})$ clouds are responsible for causing extreme weather conditions; these clouds intense convection causes the formation of Mesoscale Convective Systems (MCS) (Liu et al., 2014). A MCS is a convective system' $C b$ cloud that produces a contiguous precipitation area $\geq 100 \mathrm{~km}$ in at least one direction (Houze, 2004).

SCM pose a serious risk to aviation and may impact crops and urban populations because rapidly changing weather on various spatial and temporal scales may occur within and near $C b$ clouds (Liu et al., 2014). A large number of MCS is located in Colombia, which according to data from Tropical Rainfall Measuring Mission (TRMM) during the period 1998-2002, contribute approximately $70 \%$ of annual precipitation (Sakamoto et al., 2011). In this country disasters caused by heavy precipitation produced by SCM, hit much of the country between the second half of 2010 and the first half of 2011, causing devastating effects on the population and on local, regional and national economy (Colombia Humanitaria, 2010).

Observations of SCM are an important source of data for assimilation in weather forecasting and for monitoring climate trends (Liu et al., 2014). In this regard, images of the Geostationary Operational Environmental Satellites (GOES) have shown a high potential for detection and monitoring of SCM (Goyens et al., 2012).

A recent study investigated the possibility to improve precipitating objects detection over the Colombian continental territory from five meteorological channels rather than a single IR channel (Ramírez and Lizarazo, 2014). That study was conducted to detect and extract precipitating objects from a series of GOES13 meteorological image sub-scene. Such study applied three machine learning algorithms including support vector machine (SVM), random forest (RF) and decision tree (DT) and compared them with the infrared brightness temperature threshold (IR-BT)

\footnotetext{
${ }^{*}$ Corresponding author
}

method. Accuracy assessment was conducted using STEP (Lizarazo, 2014) using a set of NASA Tropical Rainfall Measuring Mission (TRMM) images as reference.

The results of that study suggested that the machine learning DT is more accurate for detection of precipitating objects, compared to the conventional method IR-TB, while that SVM and RF results may not be acceptable for practical applications. The DT algorithm yielded a classification model tree which exhibits a very simple structure, with only two partitions, selecting thermalinfrared (TIR1) and water vapour (WV) as the most important channels.

In contrast to such study, this article aims to: establish whether it is possible to have a classification model that can be used repeatedly, and not requiring visual interpretation of imagery for every cloudy day. The evaluation was performed for the driest day and the wettest day of 2014 and 2015. In this case, a set of Global Precipitation Measurement (GPM), a well-established satellite weather radar system, with higher resolution than TRMM was used as reference.

\section{DECISION TREE CLASSIFICATION MODEL}

DT decomposes the space's attributes in P disjoint sets, $K_{r}$ with $n \in\{1, \ldots, P\}$, using decision rules or cuts that are orthogonal to the axes' attributes. Therefore, a decision rule can be described as a simple expression of the form $X_{i} \geq T_{i}$, o $X_{i}<T_{i}$ where $T_{i}$ is some threshold, which makes this operation easy to understand by the user (Lizarazo, 2008).

In previous study, the DT algorithm yielded a tree which exhibits a very simple structure, from a meteorological image taken by the Geostationary Operational Environmental Satellite (GOES13) for twenty-tow half-hour periods for one day in 2013. Date and time for this study were selected to ensure a similar number of class representatives at the instant on April 03, 2013 at 21:15 hours Coordinated Universal Time (UTC) (201304032115, this is the date format used in the data set) taken as reference.

This tree was obtained using the Gini impurity measure, selecting thermal-infrared (thermal-IR) and water vapour (WV) as the most 
important bands. The Gini impurity measure, is a measure of the distribution of the class' label on the node, taking values in $[0,1]$, where 0 is obtained when all the elements in a node fall into the same class. DT uses the Gini impurity measure to select the cut decision with the lowest impurity in each node (Tan et al., 2005). Formally, the impurity measure Gini given by Equation 2, for $x_{i}$ attribute with $i \in\{1, \ldots, n\}$ in the $t$ node (Khalilia et al., 2011).

$$
I\left(t_{x_{i}}\right)=1-\sum_{c=0}^{c}\left(\frac{n_{c i}}{a_{i}}\right)^{2}
$$

where $\quad n$ is the number of branches in the node $t$

$N$ is the samples' number

$n_{c i}$ is the samples' number with value $x_{i}$ belonging to class $c$

$a_{i}$ is the samples' number with value $x_{i}$ in the node $t$

The Gini index of a partition is the weighted average of the Gini impurity measure the different values of $X$ attribute, given by equation 2 (Khalilia et al., 2011).

$$
\operatorname{Gini}(t, X)=\sum_{j=1}^{j} \frac{a_{i}}{N} I\left(t_{x_{i}}\right)
$$

Figure 1 shows DT algorithm classification model obtained previously, which exhibits a very simple structure, with only two partitions. Thermal-infrared (TIR1) and water vapour (WV) channel were selected as the most important channels, and the model accuracy was $98 \%$.

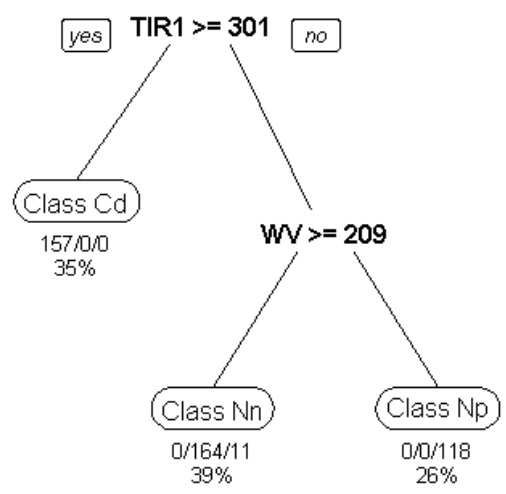

Figure 1. Decision tree obtained by the DT algorithm

The tree in Figure 1 defines the following classification rules in terms of brightness temperatures (Tb): (i) If channel TIR1 is $<$ $301 \mathrm{~K}$ then the object is Cs (Clear Sky); (ii) If channel TIR1 is $\geq 301 \mathrm{~K}$ and the channel WV is $<209 \mathrm{~K}$ then the object is $\mathrm{Nr}$ (Non-Rainfall Cloud); and (iii) If channel TIR 1 is $\geq 301 \mathrm{~K}$ and the channel WV is $\geq 209 \mathrm{~K}$, then the object is Rc (Rainfall Cloud). The $35 \%$ of training pixels fulfil the first rule, $39 \%$ the second rule and $26 \%$ the last rule. This result is similar to obtained in a previous work (Feidas et al., 2000), where the cloud classification scheme developed for the thick opaque convective clouds correspond to classes with the lower $\mathrm{Tb}$ in both the IR channel (below $223 \mathrm{~K}$ ) and WV channel (below $223 \mathrm{~K}$ ).

\section{DATA AND METHODS}

\subsection{Study area}

This study was conducted over a tropical region comprising 5062400 $\mathrm{km}^{2}$, with predominance of Colombian territory, which is located in the northwest of South America as shown in the dashed window of Figure 2a. s

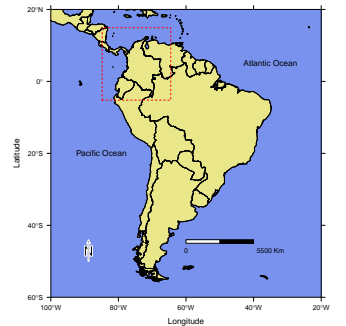

(a)

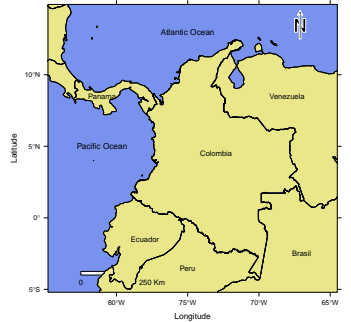

(b)
Figure 2. The study area:(a) South America. (b) Colombia.

\subsection{Data}

Meteorological images comprise a series of 88 sub-scenes in mode A (8-bits), taken by the Geostationary Operational Environmental Satellite (GOES-13) every half hour from 11:45 to 22:15 hours Coordinated Universal Time (UTC) for four different dates: (i) May 08, 2014, the rainiest day of 2014 with a precipitation of $19307.4 \mathrm{~mm}$; (ii) December 22, 2014, the least rainy day of 2014 with a precipitation of $225.9 \mathrm{~mm}$; (iii) January 06, 2015, the least rainy day of 2015 with a precipitation of $81 \mathrm{~mm}$; and (iv) October 13,2015 , the rainiest day of 2015 with a precipitation of 11900 $\mathrm{mm}$. The GOES Imager covers from visible (VIS) to thermal infrared (TIR) spectral regions with 5 spectral channels at spatial resolutions ranging from $1 \mathrm{~km}$ to $8 \mathrm{~km}$ (Table 1 ).

\begin{tabular}{|c|c|c|l|}
\hline Channel number & Central wavelength & Spatial Resolution & Channel description \\
\hline 1 & $0.52-0.72 \mu \mathrm{m}$ & $1 \mathrm{~km}$ & Visible (VIS) \\
2 & $3.78-4.03 \mu \mathrm{m}$ & $4 \mathrm{~km}$ & Mid Infrared (MIR) \\
3 & $6.47-7.02 \mu \mathrm{m}$ & $8 \mathrm{~km}$ & Water Vapor (WV) \\
4 & $10.2-11.2 \mu \mathrm{m}$ & $4 \mathrm{~km}$ & Thermal Infrared (TIR1) \\
5 & $12.9-13.8 \mu \mathrm{m}$ & $4 \mathrm{~km}$ & Thermal Infrared (TIR2) \\
\hline
\end{tabular}

Table 1. Characteristics of GOES Imager

Reference data for accuracy assessment was selected from a NASA Global Precipitation Measurement (GPM) data set, well-established satellite weather radar system, merged with high quality (IR) precipitation and monthly surface precipitation gauge data that provides half hourly consistently calibrated, uniformly gridded with spatial resolution about $10 \mathrm{~km}$ at the Equator, global precipitation data product GPM_3IMERGHH with appropriate error and metadata information (Hou et al., 2014).

\subsection{Methods}

The method proposed here comprises (Figure 3): (i) data preprocessing to convert image counts to physical quantities using the Equations 3 and 5 and match pixel size, spatial extent and spatial reference systems; (ii) pixel-based supervised cloud classification using the DT algorithm classification model obtained previously; (iii) creation of precipitating objects by binarisation and vectorization of the classified image; and (iv) accuracy assessment. The GPM precipitation amounts were also binarised, thus obtaining a set of reference precipitating objects to perform validation using STEP method (Lizarazo, 2014). STEP provides 


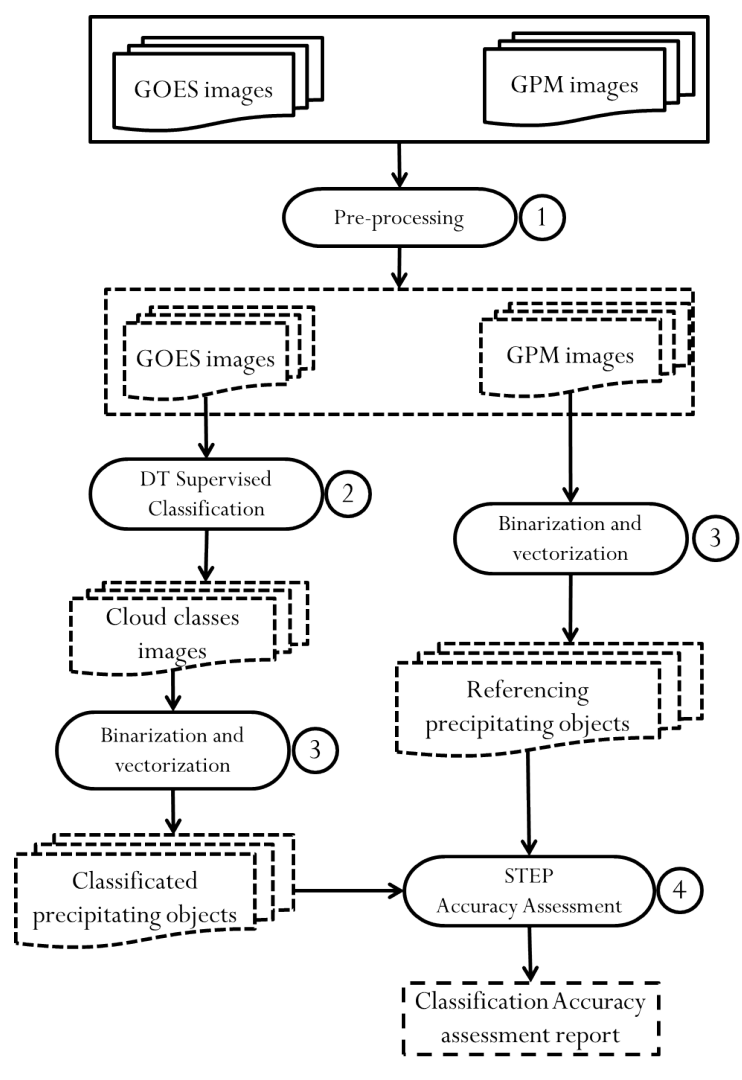

Figure 3. Methodology

four (4) similarity metrics for characterization of classified objects compared with reference objects: (i) shape similarity; (ii) theme similarity; (iii) edge similarity; and (iv) position similarity. The accuracy assessment focuses on convectives system' clouds having an area larger than $2400 \mathrm{~km}^{2}$. This threshold was chosen following a previous work (Sakamoto et al., 2011) on life cycle convective systems over Western Colombia.

Albedo (A) is calculated as:

$$
A=\left(\frac{N D_{V I S}}{255}\right)^{2}
$$

where $\quad N D_{V I S}$ are counts values in Mode-A visible channel

Brightness temperature $(\mathrm{Tb})$ is calculated as:

$$
\begin{aligned}
& T b=330 K-\frac{N D_{I R}}{2} ; \text { if } N D_{I R} \leq 176 \\
& T b=418 K-N D_{I R} ; \text { if } N D_{I R}>176
\end{aligned}
$$

where $\quad N D_{I R}$ are counts values in Mode-A infrared channel

\section{RESULTS AND DISCUSSION}

Overall accuracy was computed, similar to how overall accuracy is computed in the traditional error matrix, from shape, theme, edge and position similarity OBIA indices calculated by comparing thematic and geometric properties of classified precipitating objects with their corresponding reference precipitation objects, using a weighted area instead of the actual area for compensation of the unequal probability sampling, assigning to each classified precipitation object that matches (intersects) a given reference precipitation object a similarity value in the $[0,1]$ interval (Lizarazo, 2014).

Table 2 summarizes overall STEP similarity indices for each twentytwo date under evaluation. For the rainiest day of 2014 (20140508) the shape index presents variations in validation interval between $92 \%$ and $95 \%$, the theme index between $87 \%$ and $94 \%$, the edge index between $97 \%$ and $99 \%$ and the position index was $100 \%$ for all instants. In contrast for the rainiest day of 2015 (20151013) the shape index presents variations in validation interval between $95 \%$ and $97 \%$, the theme index between $91 \%$ and $93 \%$, the edge index between $98 \%$ and $100 \%$ and the position index was $100 \%$ between $98 \%$ and $100 \%$ for all instants.

\begin{tabular}{|c|c|c|c|c|}
\hline Day & Shape & Theme & Edge & Position \\
\hline 20140508 & {$[0.92,0.95]$} & {$[0.87,0.94]$} & {$[0.97,0.99]$} & {$[1.00,1.00]$} \\
20141222 & {$[0.94,1.00]$} & {$[0.93,1.00]$} & {$[0.99,1.00]$} & {$[0.98,1.00]$} \\
20150106 & {$[0.98,0.99]$} & {$[0.98,0.99]$} & {$[1.00,1.00]$} & {$[1.00,1.00]$} \\
20151013 & {$[0.95,0.97]$} & {$[0.91,0.93]$} & {$[0.98,1.00]$} & {$[1.00,1.00]$} \\
\hline
\end{tabular}

Table 2. Overall thematic and geometric accuracy predictors for the MCS

While for the least rainy day of 2014 day (20141222) the shape index presents variations in validation interval between $94 \%$ and $100 \%$, the theme index between $93 \%$ and $100 \%$, the edge index between $99 \%$ and $100 \%$ and the position index between $98 \%$ and $100 \%$. In contrast for the least rainy day of 2015 (20150106) the shape index presents variations in validation interval between $98 \%$ and $99 \%$, the theme index between $98 \%$ and $99 \%$, the edge index and the position index were $100 \%$ for all instants.

The results suggests that the best aspect is position, followed in their respective order by edge, shape and theme. There are no significant differences between the two dates rainiest and the two dates less rain, shown be suitable for practical applications.

A visual comparison among the time's instants under evaluation also was performed for detected cloud objects. Figure 4 shows an instant by each date under evaluation.

For the instant 201405081545 UTC (Figure 4a) of rainiest day of 2014 the overall STEP similarity was shape $95 \%$, theme $93 \%$, edge $99 \%$ and position $100 \%$, for this instant there are 21 precipitating objects which are located mainly on the Pacific, central and eastern regions of Colombia, on west region of Venezuela, on Amazon region of Brazil and on Ecuador and Peru, with areas between $1922 \mathrm{~km}^{2}$ and $117164 \mathrm{~km}^{2}$.

For the instant 201510131945 UTC (Figure 4d) of rainiest day of 2015 the overall STEP similarity was shape $96 \%$, theme $92 \%$, edge $98 \%$ and position $100 \%$, for this instant there are 55 precipitating objects which are located mainly on the Pacific, the Caribbean, central and west regions of Colombia and on Venezuela, with areas between $1005 \mathrm{~km}^{2}$ and $170715 \mathrm{~km}^{2}$.

For the instant 201412221715 UTC (Figure 4b) of least rainy day of 2014 the overall STEP similarity was shape $96 \%$, theme $97 \%$, edge $100 \%$ and position $100 \%$, for this instant there are 2 precipitating objects which are located mainly on the Colombian and Brazilian Amazon region, with areas of $3544 \mathrm{~km}^{2}$ and 11475 $\mathrm{km}^{2}$. 


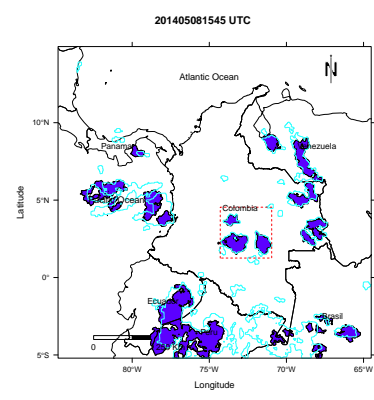

(a) 201405081545 UTC

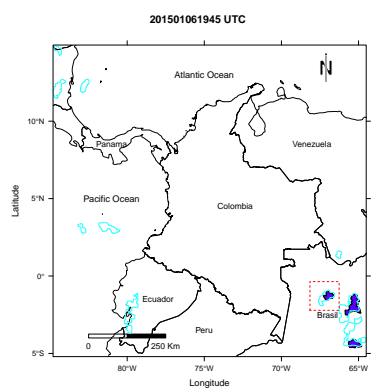

(c) 201501061945 UTC

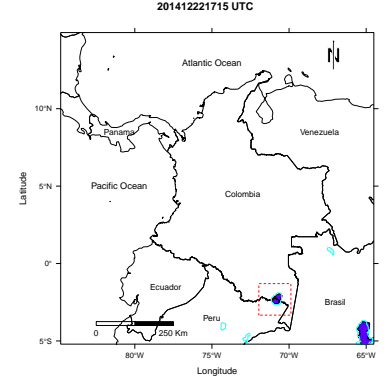

(b) 201412221715 UTC

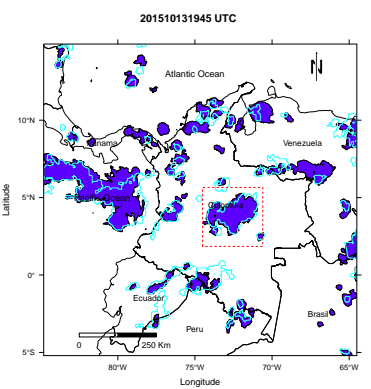

(d) 201510131945 UTC
Figure 4. Precipitating cloud objects detected at four dates. Blue colour fill represent DT classified precipitation objects, cyan colour border represent GPM reference precipitation objects and dashed red colour represent a window for analysis.

For the instant 201501061945 UTC (Figure 4b) of least rainy day of 2015 the overall STEP similarity was shape $98 \%$, theme $98 \%$, edge $100 \%$ and position $100 \%$, for this instant there are 3 precipitating objects which are located mainly on the Brazilian Amazon region, with areas between $2873 \mathrm{~km}^{2}$ and $7690 \mathrm{~km}^{2}$.

Figure 5 shows views of precipitating objects that correspond to the dashed windows in the Figure 4. In this Figure it is possible to observe that there is a strong locational match between predicted precipitation objects and reference prediction objects. However there are slight differences regarding shape and edge.

Results demonstrate how well a decision tree (DT) based classification model, inferred from a single-date GOES imagery, can be repeatedly used on different dates. This eventual transferability of empirical obtained models needs to be confirmed in more extensive experiments. However, these results suggest that machine learning techniques may be useful for better understanding meteorological phenomena as well as for improving manual methods currently used for cloud detection and tracking.

\section{CONCLUSIONS}

The main objectives of the study were accomplished: (i) evaluating the potential of using the classification model yielded by DT on many dates; and (ii) establishing that it is possible to have a classification model that can be used repeatedly for locating and tracking precipitating objects from multispectral meteorological images.

According to this study's results the best geometry aspect in the detection of precipitating objects from DT model is the position, that is, DT is able to locate accurately precipitating objects.

From the practical point of view, this work suggest a transferable decision classification model that can be used repeatedly for

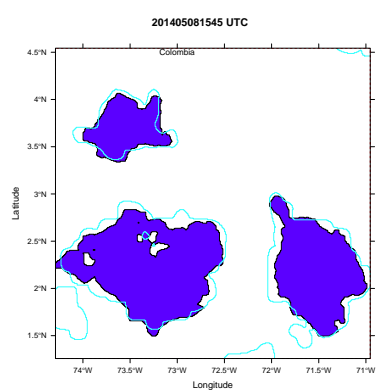

(a) 201405081545 UTC

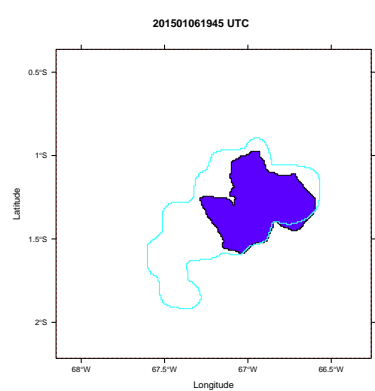

(c) 201501061945 UTC

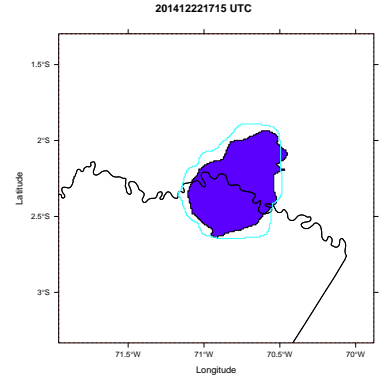

(b) 201412221715 UTC

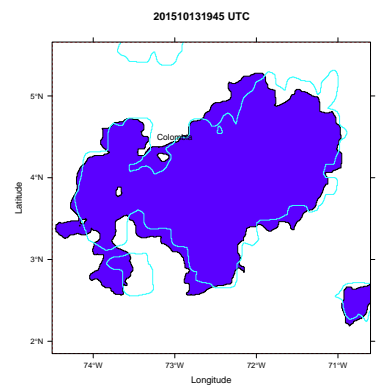

(d) 201510131945 UTC
Figure 5. Views of precipitating objects. Blue polygons represent DT classified precipitation objects and cyan edge polygons represent GPM reference precipitation objects.

locating and tracking precipitating objects from multi-temporal meteorological images, defined explicit classification rules useful for operational applications, with no visual interpretation of imagery for every cloudy day.

\section{ACKNOWLEDGEMENTS}

The authors are grateful to Instituto de Hidrología, Meteorología y Estudios Ambientales (IDEAM), the Colombian national weather service, for providing the GOES-13 meteorological images ModeA and the technical resources used in this study. The Global Precipitation Measurement (GPM) product GPM_3IMERGHH images was obtained from the National Aeronautics and Space Administration (NASA), available at http://giovanni.gsfc.nasa.gov/.

\section{REFERENCES}

Colombia Humanitaria, 2010. Reporte diario ola invernal 20102011.

Feidas, H. N., Cartalis, C. and Cracknell, A. P., 2000. Use of Meteosat imagery to define clouds linked with floods in Greece. International Journal of Remote Sensing 21(5), pp. 1047-1072.

Goyens, C., Lauwaet, D., Schröder, M., Demuzere, M. and Van Lipzig, N., 2012. Tracking mesoscale convective systems in the Sahel: relation between cloud parameters and precipitation. International Journal of Climatology 32(12), pp. 1921-1934.

Hou, A. Y., Kakar, R. K., Neeck, S., Azarbarzin, A. A., Kummerow, C. D., Kojima, M., Oki, R., Nakamura, K. and Iguchi, T., 2014. The global precipitation measurement mission. Bulletin of the American Meteorological Society 95(5), pp. 701-722.

Houze, R., 2004. Mesoscale convective systems. Reviews of Geophysics 42, pp. 1-43. 
Khalilia, M., Chakraborty, S. and Popescu, M., 2011. Predicting disease risks from highly imbalanced data using random forest. BMC medical informatics and decision making 11(1), pp. 51.

Liu, Y., Xi, D.-g., Li, Z.-1. and Shi, C.-x., 2014. Automatic Tracking and Characterization of Cumulonimbus Clouds from FY-2C Geostationary Meteorological Satellite Images. Advances in $\mathrm{Me}$ teorology.

Lizarazo, I., 2008. Clasificación de la cobertura y del uso del suelo urbano usando imágenes de satélite y algoritmos supervisados de inteligencia. UD y la GEOMÁTICA 0(2), pp. 4-18.

Lizarazo, I., 2014. Accuracy assessment of object-based image classification: another STEP. International Journal of Remote Sensing 35(16), pp. 6135-6156.

Ramírez, S. and Lizarazo, I., 2014. Digital classification of cloud masses from weather imagery using machine learning algorithms. Revista Facultad de Ingeniería Universidad de Antioquia (73), pp. 43-57.

Sakamoto, M., Ambrizzi, T. and Poveda, G., 2011. Moisture sources and life cycle of convective systems over Western Colombia. Advances in Meteorology 2011, pp. 1-11.

Tan, P., Steinbach, M. and Kumar, V., 2005. Classification: Basic Concepts, Decision Trees, and Model Evaluation. In: Introduction to Data Mining, 1st edn, Addison-Wesley, chapter Chapter 4, pp. 145-205.

Vila, D., Machado, L., Laurent, H. and Velasco, I., 2008. Forecast and Tracking the Evolution of Cloud Clusters (ForTraCC) Using Satellite Infrared Imagery: Methodology and Validation. Weather and Forecasting 23(2), pp. 233-245.

Article submitted on 10 July 2016 